\title{
Biomarker discovery, validation, standardisation $\&$ application in medical practice
}

\author{
GREIFSWALD APPROACH TO INDIVIDUALIZED \\ MEDICINE \\ - (GANI_MED) - STANDARDISATION OF CLINICAL \\ DATA FOR RESEARCH USE \\ Lorbeer $R^{1}$, Wrobel $S^{1}$, Dörr $M^{2}$, Völzke $H^{1}$, Kroemer $H K^{3}$, \\ Hoffmann $W^{1}$, Lieb $W^{1}$ \\ ${ }^{1}$ Institute for Community Medicine \\ ${ }^{2}$ Department of Internal Medicine \\ ${ }^{3}$ Institute of Pharmacology, Ernst Moritz Arndt University \\ Greifswald, Germany
}

Background: The goal of the research project Greifswald Approach to Individualized Medicine (GANI_MED) is to implement individualized diagnostic and treatment strategies using clinical routine data from our university hospital. To identify new risk factors and biomarkers of diseases, we are in the process of establishing large patient cohorts for several highly prevalent disease conditions including heart failure, stroke, renal insufficiency, and metabolic syndrome. These patient cohorts will be compared to well characterized samples from a population-based cohort study (the Study of Health in Pomerania; SHIP) in a case-control setting. One important aspect of the GANI_MED project is to use clinically obtained data for research purposes and to ensure adequate standardization and quality of these data. Aim: The aim is to standardize the routine data acquisition in the clinical context and to achieve strict quality control and standardization of these data.

Methods: The clinical characterization of the patient cohorts includes standardized questionnaires, basic medical and anthropometric examinations (e.g. blood pressure and somatometric measurements) as well as more complex procedures such as ultrasonographic imaging techniques. We developed several methods to ensure adequate standardization of the data. For the medical history, a standardized interview syntax was programmed and implemented in a mobile data capture software with data validation function using a tablet PC for medical needs. Data is stored in a data warehouse, and a medical history report is created for clinical use.

For all relevant clinical examinations, standard operating procedures (SOPs) were developed which are in line with the standards used within SHIP to ensure best possible comparability between the patient cohorts and the control sample. All physicians and technicians involved in the data acquisition process are trained, certified and supervised regularly. The obtained data are monitored closely, and in certification procedures, inter- and intra-examiner differences-as assessed by Bland-Altman-Plots-must not exceed 5\%.

For basic medical examinations including e.g. measurement of weight, height and blood pressure, identical devices across all cohorts are used. Regular device certifications, calibrations and comparative studies are performed. Individual feedback to each examiner is provided, and regular meetings with all examiners are organized on a weekly basis to ensure optimal exchange and discussions between examiners and the data quality management.

Conclusion: GANI_MED extends current research concepts of individualized medicine by in-depth standardization of clinical routine data. This will be the basis for establishing and validating individualized prevention, diagnostics and treatment strategies in our large patient cohorts.

\section{IMMUNOASSAYS IN MULTIPLEX FOR BIOMARKER DISCOVERY AND VALIDATION}

Joos TO

NMI Natural and Medical Sciences Institute at the University of Tübingen, Markwiesenstr. 55, 72770 Reutlingen, Germany

Array-based assay systems allow the determination of hundreds of molecular parameters in a single experiment. Within the last decade protein microarray technologies achieved robust analytical performance and allowed to 
screen for a multitude of parameters requiring only minimal amounts of sample material [1-4].

In this presentation the current state of protein microarrays for biomarker discovery, validation and diagnosis will be reviewed together with future demands for their application within the field of personalized medicine.

In addition, biomarker discovery activities will be discussed within the context of the FDA's critical path initiative and the European public-private "Innovative Medicines Initiative" (IMI) [5,6].

\section{References}

1. Hartmann M, Roeraade J, Stoll D, Templin MF, Joos TO. Protein microarrays for diagnostic assays. Anal Bioanal Chem. 2009; 393:1407-16.

2. Joos TO, Berger H. The long and difficult road to the diagnostic market: protein microarrays. Drug Discov Today. 2006; 11:95961 .

3. Kricka LJ, Master SR, Joos TO, Fortina P. Current perspectives in protein array technology. Ann Clin Biochem. 2006; 43(Pt 6):45767.

4. Yu X, Schneiderhan-Marra N, Joos TO. Protein microarrays for personalized medicine. Clin Chem. 2010; 56:376-87.

5. http://www.fda.gov/cdrh/ocd/criticalpath.html

6. www.imi-europe.org

THE ROLE OF LABORATORY MEDICINE IN HEALTHCARE: QUALITY REQUIREMENTS OF IMMUNOASSAYS, STANDARDISATION AND DATA MANAGEMENT IN PROSPECTIVE MEDICINE

Waerner $T^{1}$, Urthaler $J^{2}$, Krapfenbauer $K^{3}$

${ }^{1}$ Quality \& Compliance, Cell \& Molecular Biology, Boehringer-Ingelheim RCV GmbH \& CoKG, Vienna, Austria ${ }^{2}$ Process Science, In-Process-Control, Boehringer-Ingelheim RCV GmbH \& CoKG, Vienna, Austria

${ }^{3}$ Department of Cranio-Maxillofacial and Oral Surgery, Medical University of Vienna, Vienna, Austria

In the last ten years, the area of ELISA, Luminex and multiplex protein chip technology has developed and enthusiastically applied to an enormous variety of biological questions. However, the degree of stringency required in data analysis appears to have been underestimated. As a result, there are numerous published findings that are of questionable quality, requiring further confirmation and/or validation. In the course of feasibility and validation studies a number of key issues in research, development and clinical trial studies must be outlined, including those associated with laboratory design, analytical validation strategies, analytical completeness and data managements. The scope of the following präsentation should provide assistance for defining key parameters in assay evaluation and validation in research and clinical trial projects in prospective medicine.

\section{AUTOANTIBODY BIOMARKERS FOR PROSTATE CANCER DETECTION}

Klocker $H^{1}$, Massoner $P^{1}$, Ramoner $R^{1}$, Bartsch $G^{1}$, Kowald $A^{2}$, Schulz-Knappe $P^{2}$, Amersdorfer $P^{2}$, Müllner $S^{2}$, Lueking $A^{2}$

${ }^{1}$ Department of Urology, Innsbruck Medical University, Austria

${ }^{2}$ Protagen AG, Dortmund, Germany

Objectives: Prostate cancer is the most common cancer in men and the prevalence is expected to increase within an ageing society. Screening for elevated serum levels of prostate-specific antigen (PSA) is frequently used for early detection of prostate cancer in healthy men in order to enhance the chance of curative treatment. However, serum PSA has a low cancer specificity resulting in a false positive rate of $70 \%$ and more. Reducing the number of unnecessary prostate biopsies triggered by false-elevated PSA serum values would have a highly beneficial effect on both patients and healthcare costs in an ageing population. Methods: Using the proprietary UNIarray ${ }^{\mathbb{B}}$ technology we initiated a study to identify autoantigen markers that distinguish between prostate cancer and healthy men. This panel was further verified employing protein microarray chips.

Results: Stratified patient samples were incubated with the currently largest collection of recombinant human proteins available for screening purposes to detect autoantibodies against specific targets. Using plasma samples from 20 prostate cancer (pre-surgery samples, Gleason score of resected prostate: 7-9) and 20 age-matched samples of nondiseased men (PSA: $0.1-0.68 \mathrm{ng} / \mathrm{ml}$ ) we identified more than 150 prostate cancer candidate autoantigens. These autoantigen proteins were subsequently purified and printed on a protein microarray. For a first verification the protein biochip was used to test plasma or serum samples of 40 prostate cancer patients (Gleason score $>7$ ), 40 age-matched men with elevated PSA and negative biopsy (mostly diagnosed as mainly benign hypertrophy \& prostatitis) and 40 age-matched control men with normal PSA over several years. Bioinformatic analysis using different methods such as support vector machine (SVM) algorithms, classification trees, neuronal nets and a quantitative threshold method combined with 10 -fold cross validation were used to select the most promising candidates for further technical development. A subset of the marker candidates on the biochip prototype already discriminates prostate cancer patients from persons with elevated PSA and biopsy negative with a diagnostic specificity of $73 \%$ and a sensitivity of $68 \%$. A multicenter validation study is in progress.

Conclusion: Serum autoantibodies are promising biomarker candidates for improvement of specificity of prostate cancer detection. 
SYSTEMATIC DEVELOPMENT OF NOVEL ANTIBODY BIOMARKERS FOR DIAGNOSTIC PROTEIN Lueking $A^{1}$, Kowald $A^{1}$, Bartsch $G^{2}$, Klocker $H^{2}$, Göhler $H^{1}$, Amersdorfer $P^{1}$, Muellner $S^{1}$, Schulz-Knappe $P^{1}$

${ }^{1}$ Protagen AG, Dortmund, Germany

${ }^{2}$ Dept. Of Urology, Innsbruck Medical University, Austria

Prostate cancer is the most common cause of lethal cancer in men and the prevalence is expected to increase within an ageing society. Screening for elevated serum levels of prostate-specific antigen (PSA) and/or digital rectal examination (DRE) is frequently used for early detection of prostate cancer. Elevated PSA levels and/or positive DRE result trigger prostate biopsy to confirm cancer in suspect cases. Prostate biopsy is an inconvenient procedure which is associated with surgery-related risks and does not confirm prostate cancer in up to $70 \%$ of cases or more. This leaves patients with an uncertainty about a possible disease and causes further examinations including further biopsies. Reducing the number of unnecessary prostate biopsies would have a highly beneficial effect on both patients and healthcare costs in an ageing population. Consequently, for differentiation between benign prostate disease (mainly benign hypertrophy $\&$ prostatitis) and malign prostate cancer, we are developing a protein biochip for a serumbased assay with a novel set of diagnostic markers discovered using the proprietary UNIarray ${ }^{\circledR}$ technology. Briefly, stratified patient samples were incubated with the currently largest collection of recombinant human proteins blotted on PVDF membranes for screening purposes to detect autoantibodies against specific targets. Using plasma samples from 20 prostate cancer and 20 age-matched controls we have identified more than 150 prostate cancer specific candidate autoantigens. All autoantigen candidates detected in patient samples were recombinantly expressed, purified and printed on a protein microarray.

For a first verification of these novel markers the protein biochip was used to test 40 plasma samples of prostate cancer patients (Gleason score $>7$ ), 40 plasma samples of age-matched persons with elevated PSA and biopsy negative (mostly diagnosed as mainly benign hypertrophy \& prostatitis) and 40 plasma samples of age-matched control persons with normal PSA over several years as well as normal DRE. Bioinformatical methods such as support vector machine (SVM) algorithms, classification trees, neuronal nets and a quantitative threshold method combined with 10-fold cross validation are used to select the most promising candidates for further technical development. A subset of the marker candidates on the biochip prototype already discriminates prostate cancer patients from persons with elevated PSA and biopsy negative with a diagnostic specificity of $73 \%$ and a sensitivity of $68 \%$.
Conclusion: The UNIarray ${ }^{\circledR}$ technology platform comprises the systematic identification of autoantibody signatures in the serum or plasma of patients. These results may be used for e.g. patient stratification in clinical studies, treatment selection, therapy monitoring, and disease progression testing or diagnostic purposes. A verification study of the prostate cancer marker candidates with $>500$ samples is ongoing.

\section{MULTIPLEX $X$ MAP ANALYSIS-ANALYTICAL TOOL FOR PERSONALISED MEDICINE}

Vrzalová J, Topolčan $O$

Immunoanalytic laboratory, Faculty Hospital and Faculty of Medicine in Pilsen, Charles University in Prague, Czech Republic

Rapidly developing and expanding multiplex immunoanalysis opens door for precise and more adequate study of complex processes of physiology and pathophysiology. Cytokines, chemokines and growth factors are excellent candidates for multiplexed analysis because of their complex interrelationships and their complex effect on organism.

We have established the assessment of cytokines, angiogenic factors, adhesive molecules, hormones, tumour markers and other biomarkers in various biological materials of human, mouse and rat origin by multiplex immunoanalysis in our laboratory. Clinical application of this method is very wide. xMAP technology is based on binding of studied proteins to the antibodies linked to microspheres with internal spectral code referring to protein identity. The amounts of the bound proteins are determined by a second antibody connected with fluorescent molecule. The measurement is performed on special flow cytometer (luminex instrument), which determines the spectral code of microspheres - the identity of proteins - after an excitation by first laser and after an excitation by second laser it detects the amount of second antibodies on microspheresthe quantity of protein in sample. The concentrations of proteins are assessed according to standard calibration curves assayed with unknown samples.

Because of the complex nature of the method, it is important for an investigator to plan precisely the multiplex study before the application. There are three main questions:

1. Which blood sample type is adequate?

2. A number of proteins that is possible to analyse in one well?

3. Is the sensitivity of the method sufficient for all proteins measured?

Currently multiplex is mainly the task for the research field but introduction of this method in clinical use can be expected in the near future, because of its advantages: small sample volume used, complex information on the markers received 
and potentially low costs at application. However, before the use of multiplex inflammatory biomarkers analyses in clinical practice, the essential step is to standardise the pre-analytical and analytical rules and normal ranges.

Support: This study was supported by the research project VZ MSM 0021620819.

\section{THE "4S" CONCEPT: HOW TO AUGMENT THE PREDICTIVE VALUE OF PRECLINICAL RESEARCH}

Moiseeva EV, Semushina SG, Bojenko VK

Institute of Bioorganic Chemistry, Moscow, Russian Federation

The evident achievements of modern experimental medicine conceal the recognizable signs of a coming collapse: many preclinically efficient drugs demonstrated no efficacy in clinical trials. Thus, abundant resources (funds, animals, scientific potential) are spent to produce findings with no clinical value. This compels us to replace the current paradigm of preclinical research (based on randomization and averaging, and unilateral rodent models) with a new concept (multilevel set of complementary models, personalized animal data based on predictive markers, stratified sampling and subgrouping within control and experimental groups to reveal both positive and negative effect of a drug in each experiment). This personalized approach originated from our anti-breast cancer drug testing practice [1] and is currently described by the " $4 \mathrm{~S}$ " concept:

\begin{tabular}{ccc}
\hline & Personalized concept & Standard paradigm \\
\hline $\mathrm{S} \quad \begin{array}{c}\text { Set } \text { of complementary models (including } \\
\text { spontaneous and non-SPF ones) } \rightarrow\end{array}$ & Unilateral models \\
& $\begin{array}{l}\text { Multilevel models } \\
\text { S } \quad \begin{array}{c}\text { Stratification (representatives from sensitive, } \\
\text { resistant, and intermediate clusters are }\end{array}\end{array}$ & Randomization \\
& $\begin{array}{l}\text { used to generate stratified sampling) } \\
\text { Subgrouping approach to analyze data }\end{array}$ & Averaging \\
$\mathrm{S} \quad \begin{array}{c}\text { Stages of testing } \rightarrow \text { both direct and } \\
\text { long-term effects are studied in vivo } \\
\text { during consecutive steps }\end{array}$ & $\begin{array}{c}\text { Study of direct } \\
\text { drug effect }\end{array}$ \\
& & \\
\hline & &
\end{tabular}

We created three original inbred mouse strains (RB-strains, carriers of $\mathrm{Rb}$ (8.17)1Iem Robertsonian translocation) with various but high incidences of spontaneous (naturally arisen) mammary cancer (MC) rarely associated with ovary/uterus cancer and/or leukemia/lymphoma. Set of inbred strains with high (RB-strains), low (BALB/ cJCitMoise), and intermediate (A/WySnJCitMoise) inciden- ces of spontaneous $\mathrm{MC}$ and collection of transplantable tumour cell lines, including various types of $\mathrm{MC}$, T- and Bcell lymphoma/leukemia are maintained. Special documentation system permits to follow each laboratory mouse during the whole it's lifespan individually (as a patient). Newly developed classification of murine MC allows to disclose the similarities with human breast cancer pathology. Our anticancer drug testing procedure commonly follows consecutive steps from in vitro (stage 0 : sensitivity of cancer and immune cells are tested separately) to in vivo: transplanted (stage 1: preliminary tests to discover proper dosage, application time, et cetera using aged mice from non-SPF colony as recipients of tumor cells from spontaneous MC), spontaneous (stage 2: therapeutic application in females with/ without $\mathrm{MC}$ extirpation), and precancerous (stage 3: preventive regimen in females before spontaneous $\mathrm{MC}$ occurrence). Several experimental data may illustrate the advantages of our concept to be in line with personalized/predictive/ preventive holistic medicine. We collected intravital blood samples from intact mice and showed that some laboratory clinical parameters predicted (1) duration of subclinical period of MC and correlated with a stage of tumour growth in transplanted models; (2) time of spontaneous MC detection in precancerous females of RB-strains (additionally, trace element markers were used); and (3) both beneficial or non-beneficial effects of immunotherapy by local interleukin-2. Importantly, modern statistical approaches (including artificial neural networks) were used to construct relevant mathematical models.

In summary, we propose the statements of personalized concept as a few achievable steps to improve experimental research quality and predictability.

\section{Reference}

1. Moiseeva E. Anti-breast cancer drug testing. Original approaches. Novel set of mouse models. Lap Lambert academic publishing, 210 pp., 2009.

\section{KNOWLEDGE TRANSFER AND PATENTING STRATEGY IN PREDICTIVE MEDICINE \\ Walkenhorst $J$ \\ PROvendis GmbH, Mülheim/Ruhr, Germany}

Several trends have evolved over the last years which have facilitated knowledge transfer in predictive medicine from academia to industry, e.g.:

(i) A strong need to find new sources of innovation in the pharmaceutical industry in order to avoid "pipelines drying up"- softening the "not invented here"-argument 
(ii) Personalized medicine will gain more importance

(iii) Pharmaceutical industry becomes more scienceoriented. In fact, it even becomes more "academic" in structure e.g. establishing "Centers of Excellence"

(iv) Academia becomes more "business-like" with an increasing need for third party funding and a redefinition of the "mission of the university".

Knowledge transfer can occur in many different ways. However, this paper will focus on the transfer of technologies. Technology transfer offices (TTOs), which comprehensively were established in most countries not before the turn of the millennium, have demonstrably gained (more) maturity. In particular the life sciences have profited there from.

This contribution investigates current trends in technology transfer regarding predictive medicine, focusing on biomarker-licensing. It further outlines different concepts for improvements along this line.

Licensing as well as patenting activities have taken off in most industries, including life sciences. Also, biomarkers from all relevant areas of "-omics" are per se patentable, thus fulfilling an important prerequisite for commercialization.

Accordingly, the number of respective licensing agreements is swiftly increasing (as of April $28^{\text {th }}$ Medtrack $^{\mathrm{TM}}$ lists 455 agreements relating to biomarkers).

Typical agreements relate to biomarker discovery and codevelopment but also to specific biomarkers(-sets). A recent example is the Takeda-Zinfandel agreement covering TOMM40.

Most agreements are concluded between biotech and pharmaceutical companies. However, academic research institutions are a rich source of biomarkers which is reflected in the number of invention reports e.g. at PROvendis. Indeed, several licenses have been signed and there continues to be multitudinous negotiations between academia/TTOs and industry. Several biomarkers which have been identified in academia are now being marketed, including e.g. the multigene-panel of MammaPrint ${ }^{\mathrm{TM}}$.

Yet, the path of a biomarker from the academia to industry seems to be particularly winding.

To some degree this is misleading. Biomarkers are relevant for different steps in drug development and application. Inter alia, biomarkers are essential for studying mode of action of drug candidates. In this case, a detailed validation of a biomarker (by numerous independent scientific studies) will be more important to the industry than a monopoly position. Thus, although no license agreements will be signed, academia contributes enormously to drug development.

To some degree this is due to difficulties of establishing approved diagnostic biomarkers on the market as (often) significant efforts will be necessary convincing physicians and providers of other (allegedly competing) diagnostic tools that there will be a win-win-situation for all stakeholders.

To a large extend, however, reasons can be found within academia. These include the difficulty of conducting/ funding large scale studies with sufficient statistical power according to clinical development standards (however, initiatives, such as the German National Biomarker database, look promising). They also include more general issues of the present technology transfer landscape such as an ongoing need to educate scientists.

Specialised Session: Role of Laboratory Medicine in PPPM

\section{ROLE OF LABORATORY TESTING OF POLY- MORPHIC ALLELE VARIANTS OF GENE CYP2C9 OF ISOENZYME 2C9 OF CYTOCHROME P450 IN PREDICTIVE, PREVENTIVE AND PERSONAL- ISED MEDICINE ON EPILEPTOLOGY}

\section{Pilugina M, Dmitrenko D, Shnayder N}

Krasnoyarsk State Medical University named after Prof. V.F. Vojno-Jasenetsky, Krasnoyarsk, Russian Federation

Epilepsy is a widespread socially important chronic disease of brain which is treated for many years but $30 \%$ of the patients with epilepsy should take antiepileptic drugs for the life term. Client-centred approach in case of epilepsy drug therapy include assessment of the factors which influence the interand individual variability, in particular pharmacokinetic parameters changes which are due to the patient's physiologic and pathologic characteristics. In the present contribution an overview about our present knowledge how polymorphic genes can influence the VPA drugs efficacy is provided. Some emphasis will be given to different forms of cytochrome P450 which are of importance for VPA metabolism.

The purpose of the research is the assessment of the role of CYP2C9 gene polymorphisms of the isoenzyme 2C9 of cytochrome $\mathrm{P} 450$ of the liver as unmodified risk factor of adverse drug events development in case of intake of an average therapeutic doses of VPA drugs at patients with epilepsy and epileptic syndromes.

Materials and methods: Sampling included 41 cases (patients with epilepsy and epileptic syndromes). Patients were from 1 up to 60 years of age, the median was 23 years. Methods: analysis of doses of VPA preparations, TDM of VPA level in serum, study of readings in biochemical blood analyses (level of aspartate aminotransferase-AsAT, alanine aminotransferase - AlAT, bilirubin, amylase, complete albumen), illustrating functional activity of liver; video-EEGmonitoring; pharmacogenetic testing of polymorphisms of 
gene CYP2C9 (chromosome10q24.1-24.3) of isoenzyme 2C9: wild-type allele variant $\mathrm{CYP} 2 \mathrm{C} 9 * 1$ without mutation, mutant-type allele variants (CYP2C $9 * 2$ - single nucleotide replacement of cytosine by thymine in the position 430; CYP2C9*3 - single nucleotide replacement of adenine by cytosine in position 1075). Blood sampling, picking out of DNA and molecular-genetic studies were performed after a patient had given a documentary confirmation to be followed up and for filling in a patient's case record which is composed in accordance with the aim and tasks of the research.

Results: All the carriers of mutant polymorphous allelic variants CYP2C9*2 and CYP2C $9 * 3$, both homozygous and heterozygous carriers and also in case of their combination (for example, genotype CYP2C9*2/CYP2C9*3) had 100\% occurrence of treatment-emergent adverse events in case of standard usage of VPA drug dosage in accordance with the Pharmacopeia (20-30 mg/kg/per day for children, $20 \mathrm{mg} /$ $\mathrm{kg} /$ per day for adults) even during the daily dose titration (10-15 mg/kg/per day for children, $5-10 \mathrm{mg} / \mathrm{kg} /$ per day for adults). The homozygous carrier of the gene CYP2C9 of the minor allelic variant of the isoenzyme 2C9 of cytochrome $\mathrm{P} 450$ had the most severe adverse drug reactions in the form of epileptic seizures aggravation, cognitive and behavioral disorders in case of standard usage of VPA drug dosage ( $\mathrm{kg} /$ body weight per daily).

Summary: Personal approach to the VPA drug dosage in the therapy of epilepsy with children and adult patients is justified clinically. It is of medical, social and economic effectiveness in epileptology.

\section{ACTIVITY OF ANTISEPTIC SOLUTIONS IN RELA- TION TO THEIR PHYSICOCHEMICAL PROPERTIES} Gjorgjeska B, Dimova C

Faculty of Medical Sciences, Krste Misirkov bb, POB 201, 2000 Stip, Republic of Macedonia

Knowing antiseptic activity of chemical disinfectant substances has a great practical value in Predictive, Preventive and Personalised Medicine.

It is evident that there is the need for defining standard technique for quantitative determination of bactericidal activity of chemical disinfectant substances, as well as the need for defining parameter for comparing various chemical disinfectants. Solution of phenol $(5 \%)$ was considered as referent standard for evaluation of efficacy of disinfectant aqueous solutions. Suitability of phenol coefficient for evaluation of nonphenolic disinfectants is still opened question. On the other side the methods for evaluation of antiseptic activity of disinfectant aqueous solutions are microbiological.
The aim of this study is to develop a new empirical coefficient which is capable to express the various physicochemical properties of disinfectant solutions on bactericidal activity. The basic duty of this parameter (Disinfection Activity Coefficient of Solution-DACS) is to express capability for comparison and prediction of disinfectant activity. The DACS index, which is the sum of four terms (fluidity, surface tension, redox potential and osmolality), results in good correlation with the activity at different disinfectant aqueous solutions. The DACS index can be calculated using additive and statistical models. Statistical model is adequate for evaluation of different disinfectant solutions because of better expressing the bactericidal activity then additive model. For analyze of various dilutions of one disinfectant there is no significant difference between this two models. The usefulness of DACS is demonstrated for analyze of bactericidal activities on different disinfectant solutions containing boric acid, chlorhexidne, chlorhexidine with cetrimide, chloroxylenol, chlorophen, eosin, hydrogen peroxide, phenyl mercury borate, povidon-iodine, thiomersal, tosilchloramide and phenol. Results of bactericidal activities obtained from microbiological tests on Staphylococcus aureus was compared with activities predicted through DACS. In conclusion, it is considered good correlation between experimental and calculated values for bactericidal activity.

\section{REPRODUCIBILITY OF 3-PART DIFFERENTIAL HEMATOLOGY ANALYZER MEDONIC CA620: 3 YEARS OF EXPERIENCE WITH 90 000 SAMPLES IN MACEDONIA}

Ruskovska T, Kamcev N, Kamceva G, Siljanovski N

Faculty of Medical Sciences, University "Goce Delcev", Stip, Macedonia

Background: Three-part differential hematology analyzer, as a common equipment of hematology departments in routine clinical laboratories, is indispensable tool for early diagnostics and monitoring of therapy in different hematological and infectious diseases. Besides regular calibration and quality control according to good laboratory practice, a periodical check of analyzer's reproducibility is essential for getting the high quality results that contribute well to the improvement of the patients care. According to manufacturer's data Medonic CA620 hematology analyzer has the best overall reproducibility of all 3-part differential hematology analyzers currently available on Macedonian market. 
Aim of the study: The aim of our study was to determine the reproducibility of hematology analyzer Medonic CA620 after 3 years of intensive exploitation and 90000 samples analyzed. We have also compared the obtained data with results from similar study conducted after 9 months of intensive exploitation of the same analyzer and with data given from manufacturer.

Material and methods: Reproducibility of 3-part differential hematology analyzer Medonic CA620 was assessed by measuring four different fresh samples, each counted 12 times. The reproducibility of five parameters: hemoglobin (HGB), red blood cells (RBC), mean corpuscular volume (MCV), white blood cells (WBC) and platelets (PLT) was expressed as coefficient of variation (CV) of twelve measurements.

Results: For hemoglobin the following results were obtained: $13.4 \pm 0.1 \mathrm{~g} / \mathrm{dL}, \mathrm{CV}=0.75 \% ; 12.3 \pm 0.1 \mathrm{~g} / \mathrm{dL}$, $\mathrm{CV}=0.81 \% ; 10.8 \pm 0.1 \mathrm{~g} / \mathrm{dL}, \mathrm{CV}=0.93 \% ; 10.0 \pm 0.1 \mathrm{~g} / \mathrm{dL}$, $\mathrm{CV}=1.00 \%$ (mean $\mathrm{CV}=0.87 \%$ ). The results for hemoglobin reproducibility obtained from previous study were $12.9 \pm 0.1 \mathrm{~g} / \mathrm{dL}, \mathrm{CV}=0.78 \%$, and the manufacturer states for hemoglobin $\mathrm{CV}=0.60 \%$.

For RBC the following results were obtained: $5.10 \pm 0.06 \times$ $10^{6} / \mu \mathrm{L}, \mathrm{CV}=1.18 \% ; 4.86 \pm 0.05 \times 10^{6} / \mu \mathrm{L}, \mathrm{CV}=1.03 \% ; 4.51 \pm$ $0.05 \times 10^{6} / \mu \mathrm{L}, \mathrm{CV}=1.11 \% ; 3.41 \pm 0.02 \times 10^{6} / \mu \mathrm{L}, \mathrm{CV}=0.59 \%$ (mean $\mathrm{CV}=0.98 \%$ ). The results for $\mathrm{RBC}$ reproducibility obtained from previous study were $4.63 \pm 0.03 \times 10^{6} / \mu \mathrm{L}$, $\mathrm{CV}=0.65 \%$, and the manufacturer states for red blood cells $\mathrm{CV}=0.85 \%$.

For MCV the following results were obtained: $93.8 \pm 0.5 \mathrm{fL}$, $\mathrm{CV}=0.53 \% ; 87.0 \pm 0.3 \mathrm{fL}, \mathrm{CV}=0.34 \% ; 86.0 \pm 0.2 \mathrm{fL}, \mathrm{CV}=$ $0.23 \% ; 70.1 \pm 0.2 \mathrm{fL}, \mathrm{CV}=0.29 \%$ (mean $\mathrm{CV}=0.35 \%$ ). The results for MCV reproducibility obtained from previous study were $78.0 \pm 0.2 \mathrm{fL}, \mathrm{CV}=0.26 \%$, and the manufacturer states for mean corpuscular volume $\mathrm{CV}=0.50 \%$.

For WBC the results were the following: $17.2 \pm 0.2 \times 10^{3} / \mu \mathrm{L}$, $\mathrm{CV}=1.2 \% ; 12.3 \pm 0.2 \times 10^{3} / \mu \mathrm{L}, \mathrm{CV}=1.6 \% ; 6.7 \pm 0.1 \times 10^{3} / \mu \mathrm{L}$, $\mathrm{CV}=1.5 \% ; 4.6 \pm 0.1 \times 10^{3} / \mu \mathrm{L}, \mathrm{CV}=2.2 \%$ (mean $\mathrm{CV}=1.6 \%$ ). The results for $\mathrm{WBC}$ reproducibility obtained from previous study were $4.6 \pm 0.1 \times 10^{3} / \mu \mathrm{L}, \mathrm{CV}=2.2 \%$, and the manufacturer states for white blood cells $\mathrm{CV}=2.0 \%$.

Finally, for PLT the results were the following: $292 \pm 12 \times$ $10^{3} / \mu \mathrm{L}, \mathrm{CV}=4.1 \% ; 210 \pm 7 \times 10^{3} / \mu \mathrm{L}, \mathrm{CV}=3.3 \% ; 188 \pm 8 \times$ $10^{3} / \mu \mathrm{L}, \mathrm{CV}=4.3 \% ; 94 \pm 8 \times 10^{3} / \mu \mathrm{L}, \mathrm{CV}=8.5 \%$ (mean $\mathrm{CV}=$ $5.1 \%)$. The results for PLT reproducibility obtained from previous study were $233 \pm 8 \times 10^{3} / \mu \mathrm{L}, \mathrm{CV}=3.4 \%$, and the manufacturer states for platelets $\mathrm{CV}=3.3 \%$.

Conclusion: Besides the intensive 3-years exploitation and 90000 samples analyzed, the 3-part differential hematology analyzer Medonic CA620 still has an excellent reproducibility of results both for normal and pathological samples that is a robust basis for the high quality measurements in medical care, including corresponding services for preventive medicine.

\section{TECHNOLOGY PLATFORM FOR STABLE ISOTOPE LABELLING AND NON-INVASIVE ANALYSIS: METHODOLOGIES AND PROSPECTS FOR PRE- DICTIVE, PREVENTIVE AND PERSONALISED MEDICINE}

Egorova-Zachernyuk TA

Protein Labelling Innovation (PLI), BioScience Park, Archimedesweg 272333 CM Leiden, The Netherlands

Protein Labelling Innovation (PLI) is an independent research oriented technology platform company that was found by Dr. Sc. Tatiana Egorova-Zachernyuk in Leiden in 2000. PLI is based in a part of Europe well-known for its expertise in these fields. PLI's major ambitions are to develop novel technologies for the pharmaceutical and food $\&$ nutrition market. One of the goals is to contribute with the developed multidisciplinary approaches to the novel strategy in healthcare: predictive, preventive and personalised medicine (PPPM).

Core business of PLI consists of R\&D activities: cost-effective biosynthetic preparation and characterisation of biologically active molecules of various complexity, from small compounds up to complex biomolecules such as human membrane proteins, in particular drug targets. This includes labelling with stable isotopes $\left({ }^{13} \mathrm{C},{ }^{15} \mathrm{~N},{ }^{2} \mathrm{H}\right)$. PLI has unique expertise in biosynthetic preparation of high-valuable molecules from prokaryotes, archea and eukaryotes (algae, yeast, fungus, insect and mammalian cell lines).

PLI provides a proprietary technology on "Compositions and methods for stable isotope labelling of biological compounds" (PCT/NL03/00514). In November 2010 the patent is granted in Australia, AU 2004256368, and in January 2011 in Russian Federation, RU 2409657 C2. The patent application is pending in Europe, USA, Canada and Japan. PLI is offering license on the technology platform related with stable isotope $\left({ }^{13} \mathrm{C},{ }^{15} \mathrm{~N},{ }^{2} \mathrm{H}\right)$ labelling.

As an example, preparation of stable-isotope labelled drug target - human histamine H1 receptor for ssNMR structural analysis will be shown. Labelling with stable isotopes in mammalian and insect cell lines opens prospects for the preparation of high-valuable biomolecules for various applications: metabolomics, proteomics, structural biology, PPPM. Novel approaches on FT-IR rapid assessment to new sources of specific biomolecules and to the level of stable isotope enrichment will be demonstrated. In addition, rapid assessment to the level of unsaturation of fatty acids will be 
demonstrated. The technologies have a potential value to accelerate drug discovery and drug design and to develop new products for predictive diagnostics.

The PLI's technology platform opens new prospects for both, cost-effective labelling of biologically active molecules and fast non- invasive analysis, which is crucial for the development of new PPPM strategies.

Implementations and future outlook include:

- Quantification of novel diagnostics biomarkers in various branches of medicine and disease areas such as cancer, cardiovascular, diabetes, allergic, neurodegenerative diseases and other;

- Non-invasive fast screening of new sources of bioingredients and health-promoting biomolecules to be applied in functional foods and personalized therapies;
- Structure-functional insights in interactions of healthpromoting biomolecules and/or drugs with the drug targets with the aim to prevent pathologies and develop individualised strategies.

\section{References}

Egorova-Zachernyuk TA, Bosman GJ, Degrip WJ, Shvets VI. Stable isotope labelling of human histamine $\mathrm{H} 1$ receptor: prospects for structure based drug design. Dokl Biochem Biophys. 2010;433:171-174.

Egorova-Zachernyuk TA, Bosman GJ, Degrip WJ. Uniform stable isotope labelling in mammalian cells: formulation of costeffective culture medium. Appl Microbiol Biotechnol. 2011;89:397-406.

Egorova TA. Preparation and biophysical investigation of stable isotope labelled metabolites and membrane proteins: methodologies and prospects. Dr Sc. Thesis in biotechnology including bionanotechnologies (03.01.06) and in bioorganic chemistry (02.00.10), 2011. 